\title{
DENOISING OF MEDICAL ULTRASOUND IMAGES USING SPATIAL FILTERING AND MULTISCALE TRANSFORMS
}

\author{
V N Prudhvi Raj ${ }^{1}$ and Dr T Venkateswarlu ${ }^{2}$ \\ ${ }^{1}$ Associate Professor, VR Siddhartha Engineering College, Vijayawada, 520007, India \\ nagaprudhvi@yahoo.com \\ ${ }^{2}$ Professor, University College of Engineering, SV University, Tirupati, India
}

\begin{abstract}
Medical imaging became the integral part in health care where all the critical diagnosis such as blocks in the veins, plaques in the carotid arteries, minute fractures in the bones, blood flow in the brain etc are carried out without opening the patient's body. There are various imaging modalities for different applications to observe the anatomical and physiological conditions of the patient. These modalities will introduce noise and artifacts during medical image acquisition. If the noise and artifacts are not minimised diagnosis will become difficult. One of the non-invasive modality widely used is ultrasound Imaging where no question of radiation but suffers from speckle noise produced by the small particles in the tissues who's size is less than the wavelength of the ultrasound. The presence of the speckle noise will cause the low contrast images because of this the low contrast lesions and tumours can't be detected in the diagnostic phase. So there is a strong need in developing the despeckling techniques to improve the quality of ultrasound images. Here in this paper we are presenting the denoising techniques for speckle reduction in ultrasound imaging. First we presented the various spatial filters and their suitability for reducing the speckle. Then we developed the denoising methods using multiscale transforms such as Discrete Wavelet Transform (DWT), Undecimated Discrete Wavelet Transform (UDWT), dual tree complex wavelet transform (DTCDWT) and Double density dual tree complex wavelet transform (DDDTCDWT). The performance of the filters was evaluated using various metrics based on pixel based, correlation based, edge based and Human visual system (HVS) based and we found that denoising using double density dual tree complex discrete wavelet transform is outperformed with best edge preserving feature.
\end{abstract}

\section{KEYWORDS}

Discrete Wavelet Transform, Dual Tree Complex Wavelet Transform, Double density wavelet transform, double density dual tree complex wavelet transform.

\section{INTRODUCTION}

Developments in Medical imaging (X-ray's, Computed tomography, Magnetic Resonance Imaging, Ultrasound, PET and SPECT etc.) in the last few decades became helpful in diagnosis by observing the anatomical structures without opening the human body and treatment of complicated diseases such as carotid artery stenosis where a plaque is going to build up in the arteries causing the brain stroke through blocking of blood flow from heart to brain, brain tumor localisation and size of the tumor, early detection of arthritis etc.

These medical images are different from natural photographic images where the images are acquired by capturing the reflected light from the object. Here in medical imaging images are acquired for clinical procedures reflect complex physical and physiological phenomena of many different types. While forming the images every modality introduce certain amount of noise and artifacts which will complicate the diagnostic process by blurring the details and hiding the tumors etc. So the need for denoising is very important before submitting the medical images for diagnostic phase. In this paper we are concentrating on presence of noise in ultrasound medical DOI : $10.5121 /$ ijcsit.2012.4613 
images and its denoising procedures developed using spatial filtering and multiscale transforms such as discrete wavelet transform [21], Undecimated wavelet transform [25], Dual tree complex wavelet transforms [29] and Double density dual tree complex wavelet transforms [32].

The noise may be additive or multiplicative depending on the modality used for medical image acquisition. The noise due to electronic components in the acquisition hardware will be modeled with Gaussian noise which is independent of data, the data dependent noise such as quantum noise in X-ray imaging is modeled with poisson distribution, the speckle noise in ultrasound imaging is modeled with Rayleigh distribution and the noise in MRI is modeled with Rician distribution. Here in this paper we are attempting to denoise the images corrupted with speckle noise.

In ultrasound imaging the speckle noise reduces the contrast resolution of the acquired image. Because of this the detection of low contrast lesions and tumors will become difficult in diagnostic phase. The destructive interference of sound waves scattered from various sites will produce the speckle pattern in the images [1]. Scattering is a process where sound waves are forced to deviate from its path by one or more localised non-uniformities in the tissue through which they pass. These non-uniformities are called as scatterers or scattering centers. In a tissue the scattering centers arises due to inhomogeneity or small structures whose size is less than the wavelength of the ultrasound [1]. Tissue parenchyma is an example where there is a change in acoustic impedance over a microscopic level within the tissue. The scattering or speckling is caused by the tissue particles which are smaller than the wavelength of the ultrasound such as blood cells and particles that are having different acoustic impedance which are close to one another. The speckle pattern in the image is visible as light and dark spots.

The mathematical modeling of degradation and restoration process is given as

$$
\begin{aligned}
& g(x, y)=f(x, y) * h(x, y)+\eta(x, y) \\
& G(u, v)=F(u, v) H(u, v)+N(u, v)
\end{aligned}
$$

Where $g(x, y)$ is the noisy and blurred observation, $H$ is the blurring kernel and $f(x, y)$ is the signal we are recovering. In the case of denoising problem the blurring kernel will be dropped and the degradation model will be given as

$$
\begin{aligned}
& g(x, y)=f(x, y)+\eta(x, y) \\
& G(u, v)=F(u, v)+N(u, v)
\end{aligned}
$$

In the case of multiplicative noise the model is given as

$$
g(x, y)=f(x, y) \cdot \eta(x, y)
$$

Since the evolution of digital imaging many techniques were developed to remove the noise from the noisy images. Earlier the techniques were based on point processing i.e modifying the pixel intensity using some linear transformation which is not adaptive to the noise content in the image and all the pixels were processed by the same filtering rule. Due to this the filter cannot differentiate the smooth areas and edges in the image and will smooth the entire image. Because of this the edges are going to be blurred or will lose contrast so the resulting image is not an optimal solution for performing diagnostic examinations [5].

Later the neighborhood processing became popular by considering the neighboring pixel intensities while modifying the pixels intensity. In this paper we are trying to adopt the principles of these filters for the removal of speckle from the ultrasound medical images. Another major direction which we are using in this paper is multiscale transforms. These transforms decompose the noisy images into various scales and subbands and each scale and subband coefficients are processed to remove the noise from these subbands and finally the approximated image of the 
original image is reconstructed from the processed subbands. The major advantages of these transforms are energy compaction and localisation of singularities such as points, lines and edges etc.

\section{DENOISING USING SPATIAL FILTERING}

Many denoising techniques in the literature were used linear filtering based on point processing or weighted averaging of neighborhood gray levels. They will remove the noise at the expense of smoothing the edges, which are very crucial features in diagnosing the diseases from the images. Researchers Lee, Kuan, Frost etc. were proposed various denoising algorithms while considering the local statistics of the neighborhood in the filtering process. The working principle in these algorithms is computing the weighted average using sub image (neighborhood) statistics to estimate statistical measures over pixel windows varying from $3 \times 3$ up to $15 \times 15$ [6-10].

\subsection{First order statistics filtering}

The filters in this category will use the first order statistics such as mean and variance of the sub image while deciding the center pixels graylevel. The algorithms in this class will follow the following filtering equation [5]

$$
f_{x, y}=\bar{g}+k_{x, y}\left(g_{x, y}-\bar{g}\right)
$$

Where $f_{x, y}$ is the estimated noise free pixel, $g_{x, y}$ is the noisy pixel value centered in the moving window, $\bar{g}$ is the local mean value of the sub image $m \times n$ surrounding and centering the pixel $g_{x, y}, k_{x, y}$ is the weighting factor choosing the value such that $k \in[0,1]$ and $x, y$ are the coordinates of the pixel. The filtering process is controlled by the weighting factor $k_{x, y}$ and is derived in the literature by various researches as given below
i) $k_{x, y}=\frac{1-\bar{g}^{2} \sigma^{2}}{\sigma^{2}\left(1+\sigma_{n}^{2}\right)}$
ii) $k_{x, y}=\frac{\sigma^{2}}{\bar{g}^{2} \sigma_{n}^{2}+\sigma^{2}}$
iii) $k_{x, y}=\frac{\sigma^{2}-\sigma_{n}^{2}}{\sigma^{2}}$

The values $\sigma_{n}^{2}$ and $\sigma^{2}$ represents the variance of noise in the whole image and variance in the local window respectively. The noise variance is calculated as average of all variances calculated over all the windows in the whole image.

$$
\sigma_{n}^{2}=\sum_{i=1}^{p} \frac{\sigma_{p}^{2}}{\bar{g}_{p}}
$$

If the value of weighting factor is 1 (at the areas of edges) the center pixel value in the filter is unchanged to preserve the edges and when the value is zero the center pixel value in the filter is replaced with the average gray level of the neighborhood. The adaptive weiner filter uses the weighting factor in eq. $(5$, iii) to preserve the edges.

\subsection{Local Statistics filtering with higher moments}

The edge preserving nature of the filter is improved by considering the higher moment's (skewness and kurtosis) along with the mean and variance in the calculation of weighting factor as follows. In this filtering procedure the weighting factor $k_{x, y}$ in eq. (4) is replaced with window variance $\sigma_{w}^{2}$ which is called as generalized moment weighting factor [6]. 
$\sigma_{w}^{2}=\frac{c_{1} \sigma^{2}+c_{2} \sigma^{3}+c_{3} \sigma^{4}}{c_{1}+c_{2}+c_{3}}$

The constants in the above equation are calculated using the relation

$$
R=1-\frac{1}{1+\sigma^{2}}
$$

This represents the smoothness of the image. The constants $c_{1}, c_{2}, c_{3}$ are calculated by placing $\sigma^{2}, \sigma^{3}, \sigma^{4}$ in place of $\sigma^{2}$ in the above equation which is calculated from the sub image of size $m \times n$. The above equations will be applied following the condition

$c_{3} \sigma^{3} \leq c_{2} \sigma^{2} \leq c_{4} \sigma^{4}$

In the neighborhoods where the above equation is not satisfied the window variance is calculated as

$$
\sigma_{w}^{2}=\frac{c_{1} \sigma^{2}+c_{3} \sigma^{4}}{c_{1}+c_{3}}
$$

\subsection{Homogeneous mask area filtering}

The filter operates in a $[5 \times 5]$ pixel neighborhood window by finding the most homogeneous neighborhood area around each pixel using a [3 $\times 3]$ subset window as shown in the figure. The center pixel of the [5 $\times 5]$ neighborhood is substituted with the average gray level of the $3 \times 3$ mask with the smallest speckle index $C$, which is given by

$$
C=\frac{\sigma_{s}^{2}}{\bar{g}_{s}}
$$

Where $\bar{g}_{s}$ and $\sigma_{s}^{2}$ represent the mean and variance of the [3 $\left.\mathrm{x} 3\right]$ window. The window which is having smallest $C$ is the most homogeneous semi window which indicates it is not having any edge features $[7,8]$.

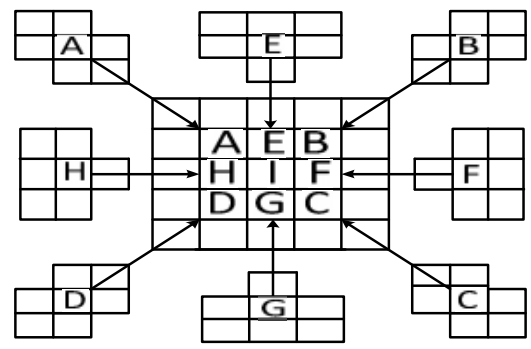

Figure 1: Homogeneous mask area filtering

\subsection{Non Linear Spatial Filtering}

The basic idea in non linear filtering is performing non linear operations on the neighborhood graylevels for selecting the graylevel of the center pixel of the neighborhood. The most popular non linear spatial filters in the literature are median filtering, maximum filtering and minimum filtering which relies on the basic principle of sorting the graylevels in the neighborhood and selecting the median, maximum and minimum value of the sorted graylevels as the graylevel of 
the center pixel in the neighborhood. In addition to the removal of noise from the images these filters introduce the ringing artifacts in the denoised images. Here we are discussing few more non linear spatial filters which will preserve the edges and minimise the ringing artifacts along with the removal of noise from the images.

\subsection{Linear scaling filters}

Here we will discuss two types of filters in this class. In the first type filter the graylevels in the neighborhood which are closer to the center pixel are selected and the mean value of this graylevels are computed. Then the center pixel in the neighborhood is replaced with this mean value. In the second type scaling filter the minimum and maximum graylevels in the neighborhood is selected and the average of these two values are computed. Then the center pixel in the neighborhood is replaced with the computed average value.

$f_{x, y}=\frac{g_{\max }+g_{\min }}{2}$

\subsection{Geometric filtering}

The geometric filtering is very much helpful in removing the speckle noise from the ultrasound images. One of the characteristic of the speckle is it appears in the image as narrow valleys (dark edges) and narrow walls (bright edges). Through the application of filter iteratively on the speckle corrupted images it will gradually fills up the dark edges i.e narrow valleys and tear down the bright edges i.e narrow walls. This filtering process will preserve the weak edges in the images. The filter operates on the image by selecting the $3 \times 3$ neighborhood with its eight neighbors. The working principle of the filter is adjusting the center pixel intensity based on the neighborhood pixel intensities. The center pixel value is incremented or decremented based on the direction and following rules to become more representative of its surroundings $[6,7,8]$.

Pixel values are assigned as shown in the above figure after selecting the direction. In this case we have selected the north-south direction first and performed the center pixel adjustments following the rules below
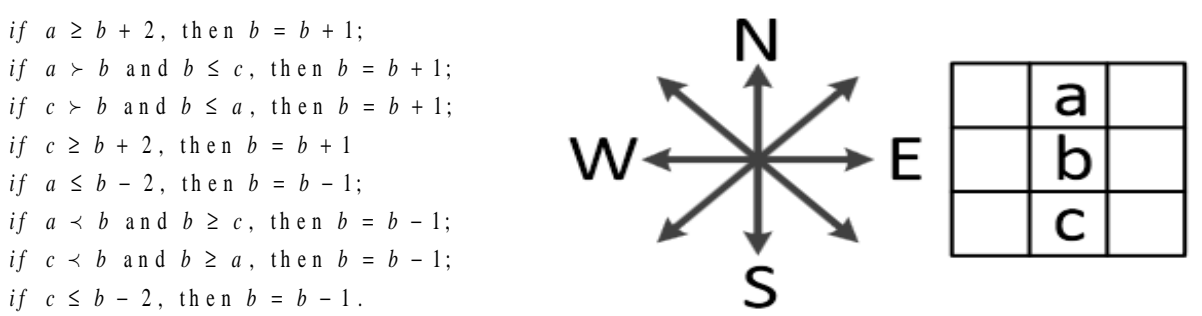

Figure 2: Geometric filtering

After performing the adgustment in the present direction perform the above step in west-east, Northeast to west-south and West-north to southeast directions. By applying the filter repeatedly we can reduce the speckle in the image.

\subsection{Maximum homogeneity over pixel neighborhood filtering}

The filter operates on the assumption that the observed area is homogeneous. It consider the $7 \times 7$ neighborhood around each pixel and estimate the homogeneity using the equation 
$f_{x, y}=\frac{C_{x, y} g_{x, y}}{\sum_{x, y} C_{x, y}}$ with $\begin{aligned} & C_{x, y}=1 \text { if }\left(1-2 \sigma_{n}\right) \bar{g} \leq g_{x, y} \leq\left(1+2 \sigma_{n}\right) \bar{g} \\ & C_{x, y}=0\end{aligned}$

As no additional parameters and thresholds are required for this filtering process the filter is suitable for automatic implementation $[8,9]$.

\section{DENOISING USING MULTISCAL TRANSFORMS}

In this section we want to use the multiscale transforms for the image denoising because they are very much useful to isolate the discontinuites present in the image and to handle the nonstationary signals or time varying signals. The spatial domain filtering discussed above is succeeded to some extent by introducing the adaptivity in the filtering scheme through first order and higher order statistics at the cost of computational cost and leaving few artifacts such as ringings and smoothing the edges. In some filters the computational cost is too high so that they are not optimal for real time filtering. To overcome these limitations lot of research was taken place in the last two decades.

The multiscale transforms such as gaussian and laplacian pyramids,steerable pyramids and wavelets are performing well in many image processing tasks by decomposing the images into multiple scales and using the benefit of sparsity and energy compaction of the above transforms that is representing the most of the information in very few coefficients. In this paper we are denoising the images using wavelet transform, undecimated wavelet transform, dual tree complex wavelet transform and double density dual tree complex wavelet transform and compared the denoising performance with various quality metrics along with observing the effect of denoising on texture of the medical images which is a very important factor while choosing the denoising algorithm.

\subsection{Discrete Wavelet Transform}

The Discrete wavelet transform of a signal $x(n)$ is computed by passing the signal through a series of filter banks. First the samples are passed through a low pass filter with impulse response $h_{0}(n)$ resulting in a convolution of the two [21]:

$$
y[n]=\left(x * h_{0}\right)[n]=\sum_{k=-\infty}^{\infty} x(k) h_{0}(n-k)
$$

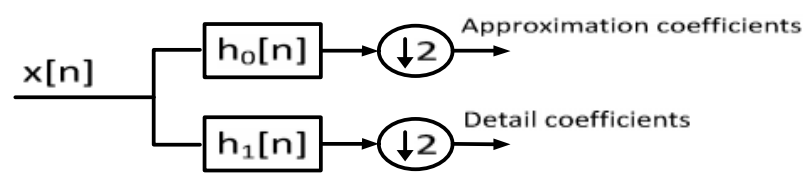

Figure 3: 1D Analysis filters

The signal is then decomposed by high-pass filter $h_{1}(n)$. The outputs of the lowpass filter are approximation coefficients and highpass filter are detail coefficients. The lowpass and highpass filters are related to each other and they form quadrature mirror filter pair. The filter outputs are then subsampled by 2 .

$$
\begin{aligned}
& y_{\text {low }}[n]=\sum_{k=-\infty}^{\infty} x(k) h_{0}(2 n-k) \\
& y_{\text {high }}[n]=\sum_{k=-\infty}^{\infty} x(k) h_{1}(2 n+1-k)
\end{aligned}
$$


2D DWT of the images can be implemented by applying 1D DWT along the rows of an image first and then applying 1D DWT on the columns of an image. When a wavelet transform is applied to an image the image is decomposed into four subbands as shown in the following figure 4 . The LL band contains the approximation coefficients, LH band contains horizontal details, HL band contains vertical details and $\mathrm{HH}$ band will contain the diagonal details [21].

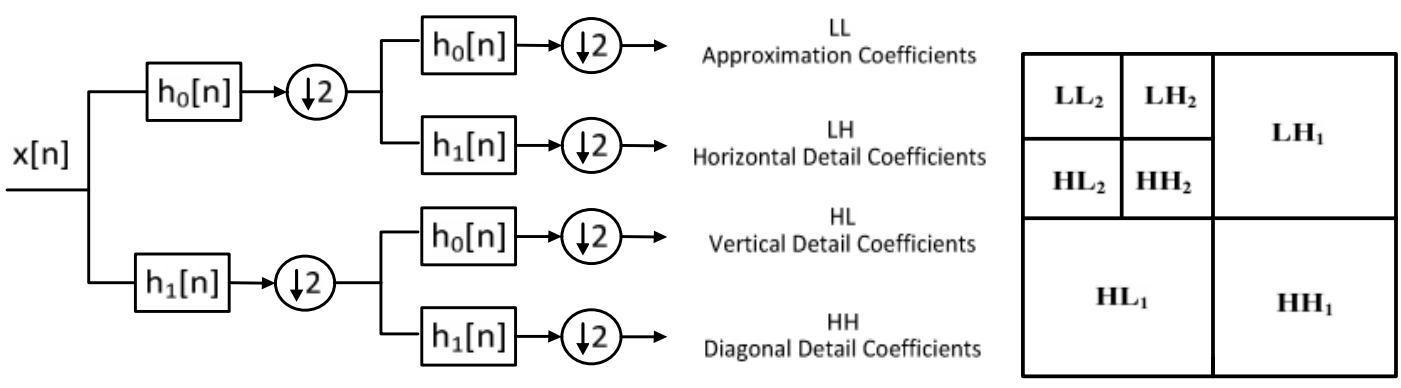

Figure 4: Wavelet filtering of an Image

The Discrete wavelet transform is suffering from four shortcomings Oscillations, Shift Variance, Aliasing, and Lack of Directionality. To overcome these shortcomings a lot of research is ongoing in present days. The Undecimated wavelet transform (UDWT) is the one of the solution by introducing the redundancy in the transform through the removal of decimation stage. The UDWT is shift invariant but it will increase the number of coefficients so the computational complexity is high.

\subsection{Dual tree complex wavelet Transform}

The performance of the UDWT is good but the redundancy introduced is very high. So the computational cost of denoising algorithm is high. To minimise this new class of wavelet transforms named wavelet frames were designed. The dual tree complex wavelet transform belongs to this category. The dual-tree complex DWT of a signal $x(n)$ is computed using two critically-sampled DWTs in parallel on the same data as shown in the following figure. If the same filters used in the upper tree and lower tree nothing is gained. So the filters in this structure were designed in a specific way that the subbands of upper DWT is interpreted as real part of complex wavelet transform and the lower tree as imaginary part. The transform is expansive by a factor 2 and shift invariant [29, 30].

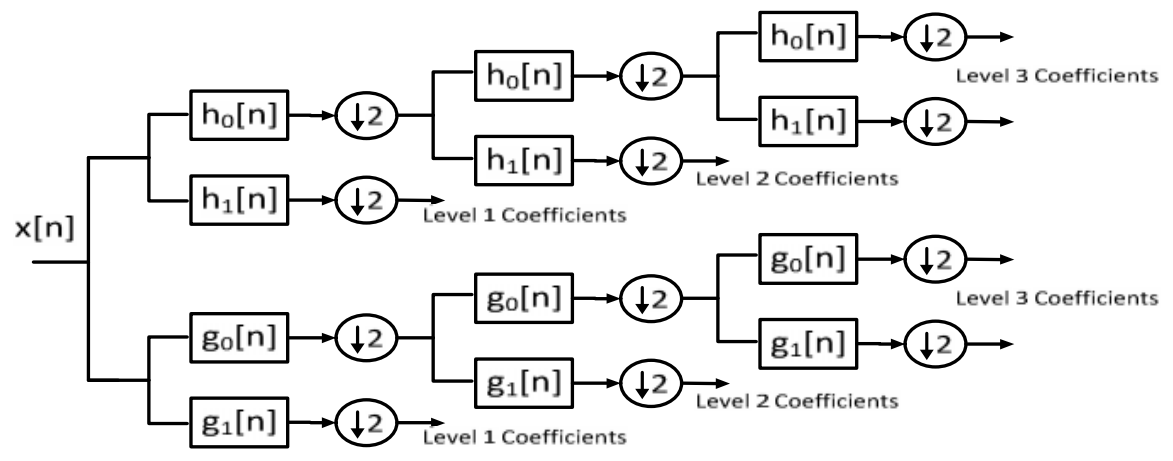

Figure 5: Dual tree wavelet (1D) filtering 
The dual tree complex wavelet transform is implemented by designing the filter banks in such a way that the filters in the second tree are the Hilbert transform of the first tree filters. That is in dual tree complex wavelet transform the filter sets in the first tree and second tree are forming a Hilbert transform pair. Let the filters $h_{0}(n), h_{1}(n)$ represents the CQF (conjugate quadrature filter) pair. That is

$\sum_{n} h_{0}(n) h_{0}(n+2 k)=\delta(k)=\left\{\begin{array}{c}1 \text { for } k=0 \\ 0 \text { for } k \neq 0\end{array}\right\}$

and $h_{1}(n)=(-1)^{(1-n)} h_{0}(n-1)$. Equivalently interms of the Z-Transform we have

$$
H_{0}^{z}(z) H_{0}^{z}(1 / z)+H_{0}^{z}(-z) H_{0}^{z}(-1 / z)=2 \text { and } H_{1}^{z}(z)=\frac{1}{z} H_{0}^{z}(-1 / z)
$$

We used the notation $H^{z}(z)$ for the z-Transform of $h(n)$ then the frequency response of the filter is $H(\omega)=H^{z}\left(e^{j \omega}\right)$. The filters $g_{o}(n)$ and $g_{1}(n)$ represent another CQF pair. Then the dilation and wavelet equations give the scaling and wavelet functions

$$
\begin{aligned}
& \phi_{h}(t)=\sqrt{2} \sum_{n} h_{0}(n) \phi_{h}(2 t-n) \\
& \psi(t)=\sqrt{2} \sum_{n} h_{1}(n) \phi_{h}(2 t-n)
\end{aligned}
$$

The scaling function $\phi_{g}(t)$ and wavelet function $\psi_{g}(t)$ are defined similarly with filters $g_{0}(n)$ and $g_{1}(n)$. For dual tree complex wavelet transforms the filters in the first tree and the filters in the second tree will form a Hilbert transform pair. $\psi_{g}(t)$ is the Hilbert transform of

$$
\begin{aligned}
& \psi_{h}(t) \text { if } \\
& \Psi_{g}(\omega)= \begin{cases}-j \Psi_{h}(\omega), & \omega>0 \\
j \Psi_{h}(\omega), & \omega<0\end{cases}
\end{aligned}
$$

Various filter design methods for dual tree complex wavelet transform were introduced by nick Kingsbury and Ivan Selesnick in their literature. The filter design is well presented in [29]. The filters must satisfy the desired properties such as approximate half sample property, Perfect Reconstruction (Orthogonal or Biorthogonal), Finite support (FIR filters), and Vanishing moments/good stop band and Linear phase. The following figure presents the wavelets in DWT and dual tree complex DWT [30].
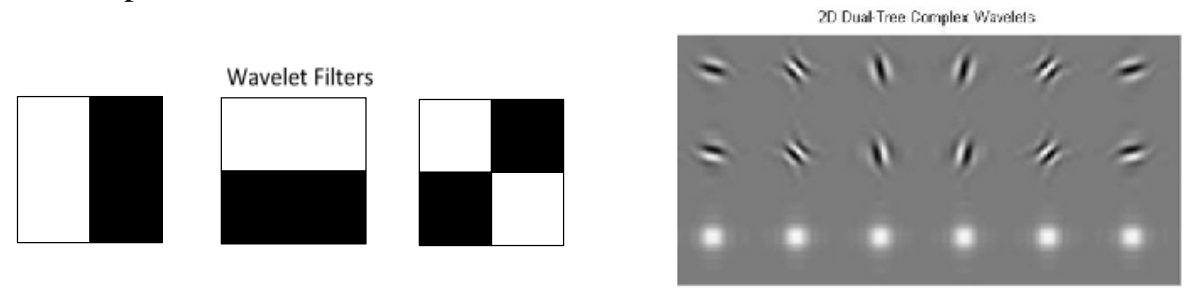

Figure 6: a) wavlet filters b) dual tree complex wavelet filters

The first two wavelets in DWT are oriented in the horizontal and vertical directions and the third wavelet cannot identify the $+45^{\circ}$ line and $-45^{\circ}$ so it mixes two diagonal orientations, which causes the checkerboard artifact. The 2D DWT is poor at isolating the two diagonal orientations i.e it can't distinguish $+45^{\circ}$ line and $-45^{\circ}$. The complex 2-D dual-tree DWT have wavelets in six distinct directions as shown in the above figure. There are two wavelets in each direction. In every 
direction, one wavelet is interpreted as real part of the complex-valued 2D wavelet, and the other wavelet is interpreted as imaginary part of the complex-valued 2D wavelet.

The complex 1D wavelet is shown in the following figure.

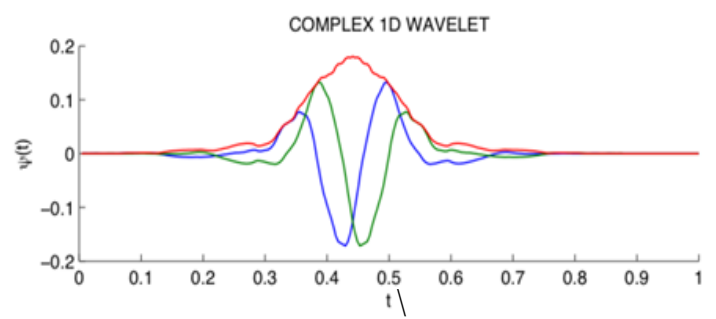

Figure 7: Complex 1D wavelet

The 2D dual tree DWT is realised by performing 1D dual tree transform along the rows of an image first and then applying 1D DTDWT on the columns of an image.

\subsection{Double Density Wavelet Transform}

Another transform belongs to the wavelet frames is Double density Wavelet transform. The transform is constructed using two distinct wavelets and a single scaling function. Within the same scale the closer spacing between adjacent wavelets can be achieved by increasing the wavelet functions. Like the dual-tree DWT, the oversampled DWT presented here is redundant by a factor of 2, independent of the number of levels. In comparison, the redundancy of the undecimated DWT grows with the number of levels.

The structure of the double density wavelet transform is shown in the following figure. It consists of one low pass filter and two distinct high pass filters represented with $h_{0}(-n), h_{1}(-n)$ and $h_{2}(-n)$ respectively. After passing through the system the signal to be analysed is processed by the low pass filter and downsampled by 2 to produce the approximation coefficients which will contain the average information of the signals. Simultaneously the signal is processed by the two distinct high pass filters and downsampled by 2 to produce the two detail coefficients. In the synthesis section the three signals are upsampled and processed by the synthesis filters which are inverse to the analysis filter to reconstruct the original signal $y(n)$. The two wavelet filters in the analysis section are designed to be offset from one another by one half- the integer translates of one wavelet fall midway between the integer translates of the other wavelet [32]

$\psi_{2}(t)=\psi_{1}(t-0.5)$

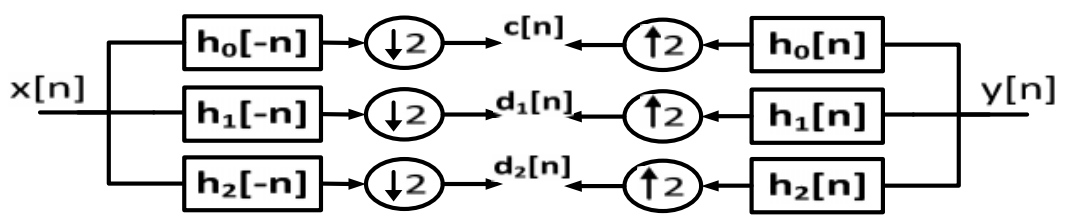

Figure 8: Double density DWT analysis and synthesis filterbank

The 2D double density DWT can be implemented by applying the 1D double density DWT to the image first along the rows and then applying along the columns. The 2D double density DWT 
Analysis filter bank is shown in the following figure. The analysis filter bank after processing the image will produce 9 subbands one of which is the 2D low pass subband and the remaining are wavelet subbands. The filters can be symmetric or asymmetric. The following tables present the filter coefficients used in this paper. The first set of filters is asymmetric whereas the second set of filters is nearly symmetric.

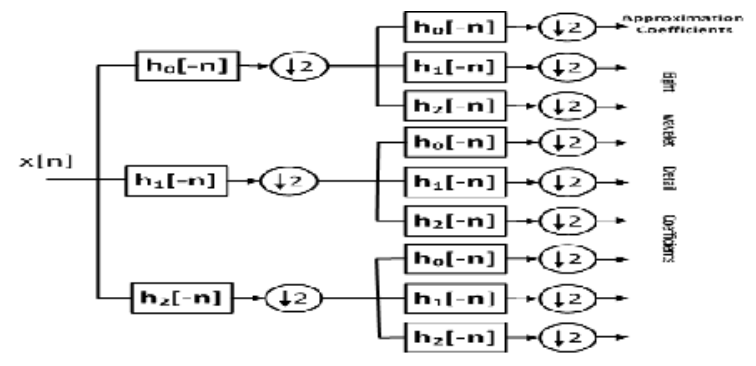

Figure 9: 2D Double density DWT analysis filterbank

Table 1: Double density DWT Filter coefficients

\begin{tabular}{|c|c|c|}
\hline $\mathbf{h}_{0}(-\mathbf{n})$ & $\mathbf{h}_{1}(-\mathbf{n})$ & $\mathbf{h}_{2}(-\mathbf{n})$ \\
\hline 0.14301535070442 & -0.01850334430500 & -0.04603639605741 \\
\hline 0.51743439976158 & -0.06694572860103 & -0.16656124565526 \\
\hline 0.63958409200212 & -0.07389654873135 & 0.00312998080994 \\
\hline 0.24429938448107 & 0.00042268944277 & 0.67756935957555 \\
\hline-0.07549266151999 & 0.58114390323763 & -0.46810169867282 \\
\hline-0.05462700305610 & -0.42222097104302 & 0 \\
\hline $\mathbf{h}_{\mathrm{a}}(-\mathbf{n})$ & $\mathbf{h}_{1}(-\mathbf{n})$ & $\mathbf{h}_{2}(-\mathbf{n})$ \\
\hline 0.00069616789827 & -0.00014203017443 & 0.00014203017443 \\
\hline-0.02692519074183 & 0.00549320005590 & -0.00549320005590 \\
\hline-0.04145457368920 & 0.01098019299363 & -0.00927404236573 \\
\hline 0.19056483888763 & -0.13644909765612 & 0.07046152309968 \\
\hline 0.58422553883167 & -0.21696226276259 & 0.13542356651691 \\
\hline 0.58422553883167 & 0.33707999754362 & -0.64578354990472 \\
\hline 0.19056483888763 & 0.33707999754362 & 0.64578354990472 \\
\hline-0.04145457368920 & -0.21696226276259 & -0.13542356651691 \\
\hline-0.02692519074183 & -0.13644909765612 & -0.07046152309968 \\
\hline 0.00069616789827 & 0.01098019299363 & 0.00927404236573 \\
\hline 0 & 0.00549320005590 & 0.00549320005590 \\
\hline 0 & -0.00014203017443 & -0.00014203017443 \\
\hline
\end{tabular}

The drawback of the double density discrete wavelet transform is chekerbaord effect i.e it can not discriminate the $+45^{0}$ and $-45^{0}$ as shown in the following figure. We have plotted the eight wavelets as grayscale images. The first two wavelets are verically oriented, the third and the sixth wavelets are horizontally oriented and the remaining four wavelets have no dominant orientation so the transform can't identify the edge features in the images effectively [32].
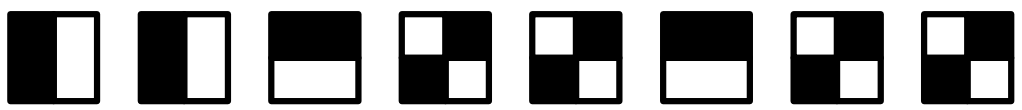

Figure 10: Double density DWT wavelet filters checkerboard artifact 
One of the solution to resolve this problem is combining the characteristics of dual tree transform and double density transform.

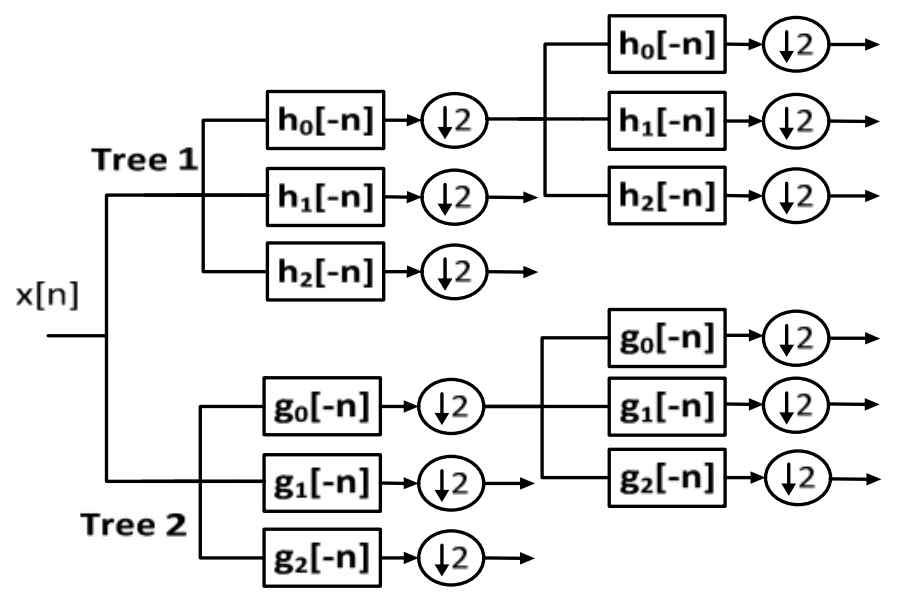

Figure 11: double density dual tree wavelet transform

The double-density complex wavelet transform is implemented by following the design rules of dual tree complex wavelet transforms.

1. The main design consideration is one wavelet pair is designed to be approximate Hilbert transforms of the other pair of wavelets

2. The second design consideration is the integer translates of one wavelet pair must fall midway between the integer translates of the other pair. This constraint can be achieved if one pair of the four wavelets is designed to be offset from the other pair of wavelets.

The design is based on two distinct scaling functions and four distinct wavelets

$$
\psi_{h, i}(t), \psi_{g, i}(t), \quad i=1,2
$$

Where the two wavelets $\psi_{h, i}(t)$ are offset from one another by one half as is $\psi_{g, i}(t)$ :

$\psi_{h, 1}(t) \approx \psi_{h, 2}(t-0.5), \quad \psi_{g, 1}(t) \approx \psi_{g, 2}(t-0.5)$

and where the two wavelets $\psi_{g, 1}(t)$ and $\psi_{h, 1}(t)$ form an approximate Hilbert transform pair as do $\psi_{g, 2}(t)$ and $\psi_{h, 2}(t)$ :

$\psi_{g, 1}(t) \approx H\left\{\psi_{h, 1}(t)\right\}, \quad \psi_{g, 2}(t) \approx H\left\{\psi_{h, 2}(t)\right\}$

The filters in this paper are designed based on the design procedure given in [32]. The detailed study on the filter design for double density dual tree complex wavelet transform can be found in [29]. The first stage filters in the implementation are different from the filters of the remaining stages in the tree. The analysis filters in the first tree will become the synthesis filters to the second tree and vice versa. The mathematical background on complex dual tree DWT is well presented in the papers $[29,30]$. The filters designed for this work from the above design procedure is given in the tables 
International Journal of Computer Science \& Information Technology (IJCSIT) Vol 4, No 6, December 2012

Table 2: Double Density Dual Tree First stage Wavelet filter Coefficients

\begin{tabular}{|c|c|c|}
\hline$h_{0}(-n)$ & $h_{1}(-n)$ & $h_{2}(-n)$ \\
\hline 0 & 0 & 0 \\
\hline 0.00069616789827 & -.00014203017443 & 0.00014203017443 \\
\hline-.02692519074183 & 0.00549320005590 & -0.00549320005590 \\
\hline-.04145457368920 & 0.01098019299363 & -0.00927404236573 \\
\hline 0.19056483888763 & -.13644909765612 & 0.07046152309968 \\
\hline 0.58422553883167 & -.21696226276259 & 0.13542356651691 \\
\hline 0.58422553883167 & 0.33707999754362 & -0.64578354990472 \\
\hline 0.19056483888763 & 0.33707999754362 & 0.64578354990472 \\
\hline-.04145457368920 & -.21696226276259 & -0.13542356651691 \\
\hline-.02692519074183 & -.136449097655612 & -0.07046152309968 \\
\hline 0.00069616789827 & 0.01098019299363 & 0.00927404236573 \\
\hline 0 & 0.00549320005590 & 0.00549320005590 \\
\hline 0 & -.00014203017443 & -0.00014203017443 \\
\hline 0 & 0 & 0 \\
\hline$g_{0}(-n)$ & $g_{1}(-n)$ & $\mathrm{g}_{2}(-\mathrm{n})$ \\
\hline 0 & 0 & 0 \\
\hline 0 & 0 & 0 \\
\hline 0.00069616789827 & -0.00014203017443 & 0.00014203017443 \\
\hline-.02692519074183 & 0.00549320005590 & -.00549320005590 \\
\hline-.04145457368920 & 0.01098019299363 & -.00927404236573 \\
\hline 0.19056483888763 & -0.13644909765612 & 0.07046152309968 \\
\hline 0.58422553883167 & -0.21696226276259 & 0.13542356651691 \\
\hline 0.58422553883167 & 0.33707999754362 & -.64578354990472 \\
\hline 0.19056483888763 & 0.33707999754362 & 0.64578354990472 \\
\hline-.04145457368920 & -0.21696226276259 & -.13542356651691 \\
\hline-.02692519074183 & -0.13644909765612 & -.07046152309968 \\
\hline 0.00069616789827 & 0.01098019299363 & 0.00927404236573 \\
\hline 0 & 0.00549320005590 & 0.00549320005590 \\
\hline 0 & -0.00014203017443 & -.00014203017443 \\
\hline
\end{tabular}

The above filters are the first stage filters in the tree 1 and tree 2 of double density dual tree discrete wavelet transform. These filters are only applied in the first stage decomposition only. The filters for the remaining stages are given below.

Table 3: Double Density Dual Tree Wavelet filter Coefficients from second stage onwards

\begin{tabular}{lll}
\hline \multicolumn{1}{c}{$\mathrm{g}_{0}(-\mathrm{n})$} & \multicolumn{1}{c}{$\mathrm{g}_{!}(-\mathrm{n})$} & \multicolumn{1}{c}{$\mathrm{g}_{2}(-\mathrm{n})$} \\
\hline 0.00005956893024 & 0.00004311757177 & -0.00037033761102 \\
-0.00813549683439 & -0.00588868840296 & 0.05057805218407 \\
-0.01971220693439 & -0.01484700537727 & 0.11428688385011 \\
0.11239376309619 & 0.16040017815754 & 0.42976785708978 \\
0.45630440337458 & 0.47089724990858 & -0.68031992557818 \\
0.59529279993637 & -0.64763513288874 & 0.01936710587994 \\
0.29533805776119 & 0.02671809818132 & 0.06840460220387 \\
0.00737700819766 & -0.00675291099550 & 0.00027261232228 \\
-0.02488304194507 & 0.01718853971559 & -0.00200122286479 \\
0.00017870679071 & -0.00012344587034 & 0.00001437252392 \\
\hline
\end{tabular}

\begin{tabular}{cll}
\hline $\mathrm{h}_{0}(-\mathrm{n})$ & \multicolumn{1}{c}{$\mathrm{h}_{\mathrm{t}}(-\mathrm{n})$} & \multicolumn{1}{c}{$\mathrm{h}_{2}(-\mathrm{n})$} \\
\hline 0.00017870679071 & -0.00012344587034 & 0.00001437252392 \\
-0.02488304194507 & 0.01718853971559 & -0.00200122286479 \\
0.00737700819766 & -0.00675291099550 & 0.00027261232228 \\
0.29533805776119 & 0.02671809818132 & 0.06840460220387 \\
0.59529279993637 & -0.64763513288874 & 0.01936710587994 \\
0.45630440337458 & 0.47089724990858 & -0.68031992557818 \\
0.11239376309619 & 0.16040017815754 & 0.42976785708978 \\
-0.01971220693439 & -0.01484700537727 & 0.11428688385011 \\
-0.00813549683439 & -0.00588868840296 & 0.05057805218407 \\
0.00005956893024 & 0.00004311757177 & -0.00037033761102 \\
\hline
\end{tabular}

\subsection{Denoising Procedure using Multiscale Transforms}

1. Compute the forward transform of the image to be denoised and decompose the image into subbands 
International Journal of Computer Science \& Information Technology (IJCSIT) Vol 4, No 6, December 2012

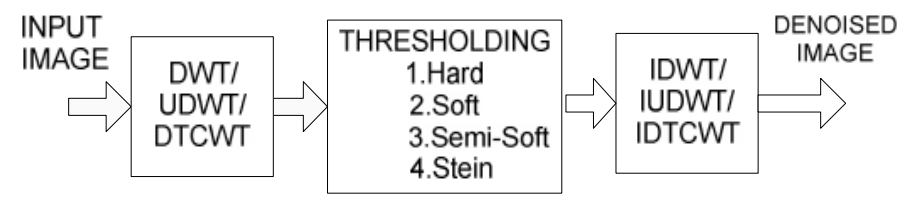

Figure 12: Denoising system using Multiscale Transform

2. Compute the threshold from the first scale HH (vertical details) band using the MAD (median absolute deviation) using the following formula considering that most of the noise is present in that band.

$$
\hat{\sigma}(\mathrm{mad})=\frac{\text { median }\left\{\left|w_{j}\right|: j=1,2, \ldots \frac{k}{2}\right\}}{0.6745}
$$

3. Apply the shrinkage step (modifying the wavelet coefficients in the subbands) using the following shrinkage rules [22, 23, 24]

Table 4: Shrinkage Rules

\begin{tabular}{|c|c|c|c|}
\hline Hard Thresholding & Soft Thresholding & & Semi-soft Thresholding \\
\hline$D_{H}^{F}(w)=\left\{\begin{array}{lc}w & \text { for all }|w|>T \\
0 & \text { otherwise }\end{array}\right.$ & $D_{H}^{s}(w)=\operatorname{sgn}(w) \max (0 ;|w|-T)$ & $D_{s s}^{m s}(w)=$ & $=\left\{\begin{array}{cc}0 & |w| \leq T \\
\operatorname{sgn}(w) \frac{T(|w|-T)}{T 1-T} & T<|w| \leq T 1 \\
w & |w|>T 1\end{array}\right.$ \\
\hline
\end{tabular}

4. After modifying the wavelet coefficients in the subbands take the inverse transform to reconstruct the image to get denoised image which is an estimation of the original one.

Many shrinkage rules are associated with the wavelet processing. The threshold may be calculated globally, level dependent or subband dependent. But here we are calculating the threshold globally. The shrinkage rules may also be changed level to level and subband to subband based on the local statistics of the wavelet coefficients at that particular level or subband. In this paper we are applying the same shrinkage rule for all the subbands and all the levels.

\section{Evaluation criteria for Denoising Algorithms}

To evaluate the quality of the image processing algorithms there are several metrics proposed in the literature. There are six categories of metrics which are used in image quality assessment they are i) Pixel difference based measures ii) Correlation based measures iii) Edge based measures iv) Spectral distance measures v) Context based measures and vi) Human visual system based measures. Here we are comparing our denoising algorithms using a group of metrics drawn from the above class and performance of the algorithms was observed.

\subsection{Pixel difference based measures}

\subsubsection{Minkowski metrics}

The $L_{\gamma}$ norm of the dissimilarity of two images can be calculated by calculating the minkowski average of the pixel differences spatially and then chromatically as given below 
$\varepsilon^{\gamma}=\frac{1}{K} \sum_{k=1}^{K}\left\{\frac{1}{M N} \sum_{x=0}^{M-1} \sum_{x, y=0}^{N-1}\left|f_{k}(x, y)-\hat{f}_{k}(x, y)\right|^{\gamma}\right\}^{\frac{1}{\gamma}}$

Where $f(x, y)$ is the reference image, $\hat{f}(x, y)$ is the estimated image of $f(x, y)$ by our denoising algorithm with the input $g(x, y)$ which is a noisy version of $f(x, y)$.

For $\gamma=1$ we obtain the absolute difference (AD), for $\gamma=2$ we will obtain the mean square error (MSE). Along with these two measures we are calculating minkowski measures for $\gamma=3$ and $\gamma=4$ in this paper to observe the performance of our algorithms.

\subsubsection{PSNR (Peak Signal to Noise Ratio)}

PSNR is the widely used pixel based measure in decibels (dB). The PSNR is computed using the following formula

$$
P S N R=20 \log _{10}\left(\frac{2^{B}-1}{\sqrt{M S E}}\right)
$$

Where B represents bits per sample and MSE (Mean Squared error) is the mean square error between a signal $f(x, y)$ and an approximation $\hat{f}(x, y)$ is the squared norm of the difference divided by the number of elements in the signal.

$$
\begin{aligned}
& M S E=\|f(x, y)-\hat{f}(x, y)\|^{2}=\frac{1}{M N} \sum_{x=0}^{M} \sum_{y=0}^{N}[f(x, y)-\hat{f}(x, y)]^{2} \\
& R M S E=\sqrt{\frac{1}{M N} \sum_{x=0}^{M} \sum_{y=0}^{N}[f(x, y)-\hat{f}(x, y)]^{2}}
\end{aligned}
$$

MSE and RMSE measures the difference between the original and distorted sequences. PSNR measures the fidelity i.e how close a sequence is similar to an original one.

\subsubsection{Maximum Difference}

Maximum difference is defined as

$$
M D=\max (|f(x, y)-\hat{f}(x, y)|)
$$

The large value of maximum difference means denoised image is poor quality.

\subsubsection{Normalised Absolute Error (NAE)}

The large value of normalised absolute error means that denoised image is poor quality and is defined as

$$
N A E=\frac{\sum_{x=0}^{M-1} \sum_{y=0}^{N-1}|f(x, y)-\hat{f}(x, y)|}{\sum_{x=0}^{M-1} \sum_{y=0}^{N-1}|f(x, y)|}
$$




\subsubsection{Signal to Noise Ratio (SNR)}

Signal to noise ratio in an image is calculated as

$$
S N R=\frac{\mu}{\sigma}
$$

Where $\mu$ is the average information in the signal and $\sigma$ is the standard deviation of the signal which represents the amount of noise present in the image. There is one more measure is there similar to the SNR it is signal to background ratio.

$$
S B R=\frac{\mu}{\sigma_{B G}}
$$

Subtract background from the image calculate standard deviation from it and finally compute the above ratio.

\subsection{Correlation based measures}

The correlation between two images can also be quantified interms of correlation function. These measures measure the similarity between the two images hence in this sense they are complementary to the difference based measures.

\subsubsection{Structural content}

For an $M \times N$ image the structural content is defined as

$$
S C=\frac{1}{K} \sum_{k=1}^{K} \frac{\sum_{x=0}^{M-1} \sum_{y=0}^{M-1} \sum_{y=0}^{N-1} \hat{f}_{k}(x, y)^{2}}{\hat{f}_{k}}
$$

The large value of structural similarity means that denoised image is poor quality

\subsubsection{Normalised cross correlation measure (NK)}

The normalised cross correlation measure is defined as

$$
N K=\frac{1}{K} \sum_{k=1}^{K} \frac{\sum_{x=0}^{M-1} \sum_{y=0}^{N-1} f_{k}(x, y) \hat{f}_{k}(x, y)}{\sum_{x=0}^{M-1} \sum_{y=0}^{N-1} f_{k}(x, y)^{2}}
$$

\subsubsection{Czekanowski distance}

A metric useful to compare vectors with strictly positive components as in the case of images is given as

$$
C=\frac{1}{M N} \sum_{x=0}^{M-1} \sum_{y=0}^{N-1}\left[1-\frac{2 \sum_{k=1}^{K} \min \left(f_{k}(x, y), \hat{f}_{k}(x, y)\right)}{\sum_{k=1}^{K} f_{k}(x, y)+\hat{f}_{k}(x, y)}\right]
$$


This coefficient is also called as percentage similarity measures the similarity between different samples, communities and quadrates.

\subsection{Edge Based metrics}

\subsubsection{Laplacian Mean Square Error (LMSE)}

This measure is based on importance of edges measurement. The large value of Laplacian mean square error means that the image is poor quality. LMSE is defined as

$$
L M S E=\frac{\sum_{x=0}^{M-1} \sum_{y=0}^{N-1}[L(f(x, y))-L(\hat{f}(x, y))]^{2}}{\sum_{x=0}^{M-1} \sum_{y=0}^{N-1}[L(f(x, y))]^{2}}
$$

\subsection{HVS based metrics}

\subsubsection{Universal Image Quality Index (UQI)}

It is a measure used to find the image distortion using three factors i) Luminance distortion ii) Loss of correlation, and iii) Contrast distortion.

If two images $f(x, y)$ and $\hat{f}(x, y)$ are considered as a matrices with $\mathrm{M}$ column and $\mathrm{N}$ rows containing pixel values $f(x, y)$ and $\hat{f}(x, y)$ respectively the universal image quality index $\mathrm{Q}$ may be calculated as a product of three components

$$
Q=\frac{\sigma_{\hat{f f}}}{\sigma_{f} \sigma_{\hat{f}}} \cdot \frac{2 \bar{f} \overline{\hat{f}}}{f^{2}+\hat{f}^{2}} \cdot \frac{2 \sigma_{f} \sigma_{\hat{f}}}{\sigma_{f}^{2}+\sigma_{\hat{f}}^{2}}
$$

Where $\bar{f}=\frac{1}{M N} \sum_{x=0}^{M-1} \sum_{y=0}^{N-1} f(x, y) \quad$ and $\quad \overline{\hat{f}}=\frac{1}{M N} \sum_{x=0}^{M-1} \sum_{y=0}^{N-1} \hat{f}(x, y)$

$$
\begin{aligned}
& \sigma_{\hat{f f}}=\frac{1}{M+N-1} \sum_{x=0}^{M-1} \sum_{y=0}^{N-1}(f(x, y)-\bar{f})(\hat{f}(x, y)-\overline{\hat{f}}) \\
& \sigma_{f}^{2}=\frac{1}{M+N-1} \sum_{x=0}^{M-1} \sum_{y=0}^{N-1}(f(x, y)-\bar{f})^{2} \text { and } \sigma_{\hat{f}}^{2}=\frac{1}{M+N-1} \sum_{x=0}^{M-1} \sum_{y=0}^{N-1}(\hat{f}(x, y)-\overline{\hat{f}})^{2}
\end{aligned}
$$

The first component in the above formula is correlation coefficient. It measures the degree of linear correlation between images. The range of this component is $[-1,1]$. The best value 1 is obtained when the images are linearly related. The second component in the formula measures luminance distortion between the images. The range of this component is $[0,1]$. The third component measures the contrast distortion between the images the range for this component is $[0,1]$. The range of values for $\mathrm{Q}$ is $[-1,1]$. The value 1 is obtained when the images are identical. 


\subsubsection{Structural similarity}

(SSIM) index is another method based on HVS for measuring the similarity between two images [28].

The SSIM metric is computed on various windows of an image. The measure between two windows $x$ and $y$ of common size $N \times N$ is [28]:

$$
\operatorname{SSIM}(x, y)=\frac{\left(2 \mu_{x} \mu_{y}+c_{1}\right)\left(2 \sigma_{x y}+c_{2}\right)}{\left(\mu_{x}^{2}+\mu_{y}^{2}+c_{1}\right)\left(\sigma_{x}^{2}+\sigma_{y}^{2}+c_{2}\right)}
$$

Where

$\mu_{x}$ is the average of $x$ and $\mu_{y}$ is the average of $y, \sigma_{x}^{2}$ is the variance of $x$ and $\sigma_{y}^{2}$ is the variance of $\mathrm{y}, \sigma_{x y}$ is the covariance of $x$ and $y, c_{1}=\left(k_{1} L\right)^{2}, c_{2}=\left(k_{2} L\right)^{2}$ two variables to stabilize the division with weak denominator, $L$ is the dynamic range of the pixel values (typically this is $2^{\# \text { bits per pixel }}-1$ ),$k_{1}=0.01$ and $k_{2}=0.03$ by default. To evaluate the image quality this formula is applied only on luminance component. The resultant SSIM index is a decimal value between -1 and 1 , and value 1 is only reachable in the case of two identical sets of data.

\section{RESULTS}

The performance of the algorithms was evaluated based on the above quality metrics obtained from the original image and the denoised image.

\subsection{Denoising using Spatial Filters}

Table 5: Performance of various spatial filters

\begin{tabular}{|c|l|l|l|l|l|l|}
\hline & Wiener & LSMV & $\begin{array}{c}\text { Homogeneous } \\
\text { mask area }\end{array}$ & $\begin{array}{c}\text { Hybrid } \\
\text { median }\end{array}$ & $\begin{array}{c}\text { Linear } \\
\text { scaling(Avg) }\end{array}$ & Geometric \\
\hline MSE & 145.1333 & 575.7459 & 499.2489 & 330.9807 & 565.4630 & 744.2902 \\
\hline SNR & 16.9359 & 10.6931 & 11.2321 & 13.1680 & 10.9319 & 11.0001 \\
\hline RMSE & 12.0471 & 23.9947 & 22.3439 & 18.1929 & 23.7795 & 27.2817 \\
\hline PSNR & 29.5234 & 23.5388 & 24.1579 & 25.9431 & 23.6171 & 22.4237 \\
\hline ME3 & 15.2842 & 32.2989 & 30.9940 & 26.8304 & 31.4119 & 41.5120 \\
\hline ME4 & 17.9389 & 39.3798 & 38.9692 & 34.7493 & 37.7734 & 54.3239 \\
\hline UQI & 0.7670 & 0.5322 & 0.6526 & 0.7671 & 0.5576 & 0.7536 \\
\hline SSIM & 0.7915 & 0.5703 & 0.6714 & 0.7812 & 0.5904 & 0.7566 \\
\hline AD & -0.0606 & 0.3213 & 2.4525 & 1.0506 & -1.6313 & -11.4211 \\
\hline SC & 1.0944 & 1.2405 & 1.2762 & 1.1952 & 1.1656 & 0.6640 \\
\hline NK & 0.9375 & 0.8261 & 0.8246 & 0.8741 & 0.8540 & 1.1535 \\
\hline MD & 61.0000 & 146.0000 & 217.0000 & 203.0000 & 119.0000 & 240.0000 \\
\hline LMSE & 0.2703 & 0.9805 & 0.8670 & 0.5545 & 0.9139 & 0.8600 \\
\hline NAE & 0.2071 & 0.3754 & 0.3399 & 0.2301 & 0.3831 & 0.3118 \\
\hline
\end{tabular}



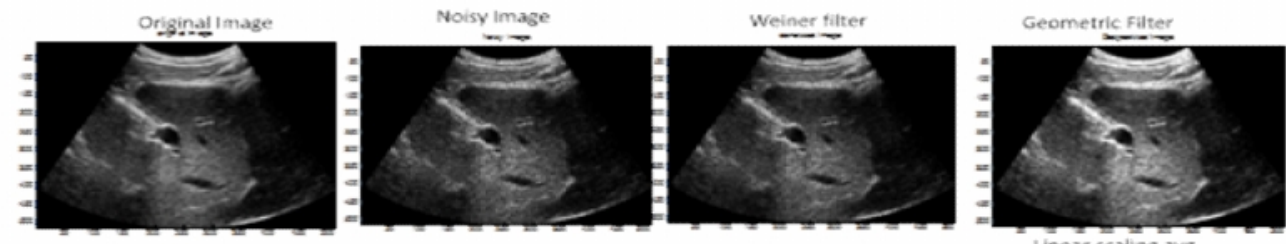

Localstotigtics my

Homogenious mask area
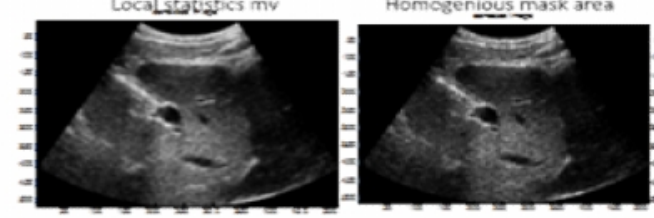

Hybrid median filter
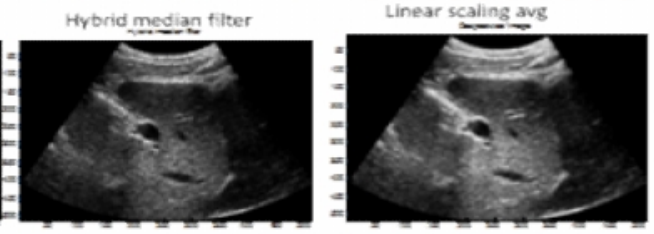

Figure 13: [Left to Right] a) Original Image b) Noisy Image c) Wiener filtering d) LSMV e) Homogeneous mask area f) Hybrid median g) Linear scaling f) Geometric filtering

\subsection{Denoising using DWT and UDWT}

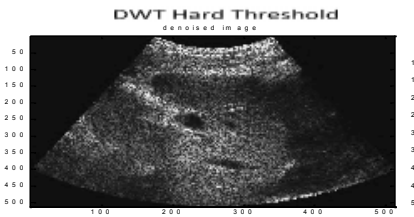

UDWT soft Threshold

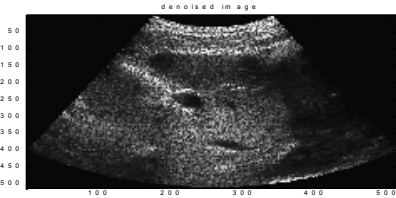

DWT soft Threshold

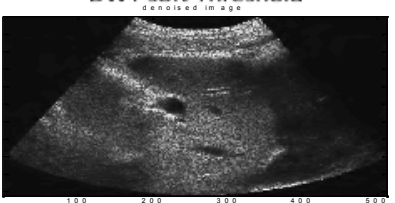

UDWT Hard Threshold

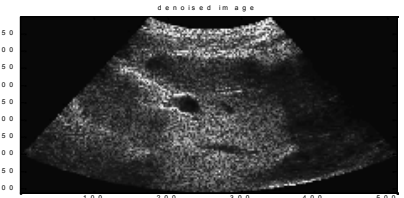

DWT semisoft Threshold

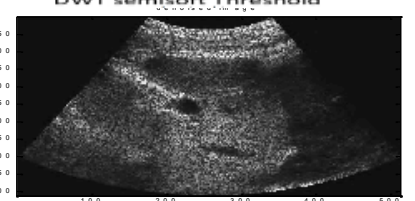

UDWT semisoft Threshold

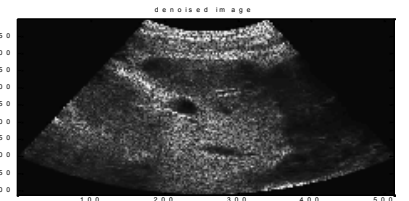

Figure 14: Denoised Results a) DWT Hard b) DWT Soft c) DWT Semi soft e) UDWT Hard f) UDWT Hard g) UDWT semisoft

Table 6: Performance evaluation of DWT and UDWT denoising

\begin{tabular}{|c|l|l|l|l|l|l|}
\hline & \multicolumn{3}{|c|}{ DWT } & \multicolumn{3}{c|}{ UDWT } \\
\hline & \multicolumn{1}{|c|}{ Soft } & \multicolumn{1}{|c|}{ Hard } & \multicolumn{1}{c|}{ Semisoft } & \multicolumn{1}{c|}{ Soft } & \multicolumn{1}{c|}{ Hard } & \multicolumn{1}{c|}{ Semisoft } \\
\hline MSE & 273.2872 & 582.8712 & 533.4604 & 541.2894 & 539.2893 & 469.6580 \\
\hline SNR & 13.7038 & 10.6961 & 11.0521 & 11.0123 & 11.0186 & 11.5688 \\
\hline RMSE & 16.5314 & 24.1427 & 23.0967 & 23.2656 & 23.2226 & 21.6715 \\
\hline PSNR & 26.3562 & 23.0666 & 23.4514 & 23.3881 & 23.4042 & 24.0046 \\
\hline ME3 & 23.9107 & 32.9436 & 32.0036 & 32.1925 & 32.0573 & 30.6391 \\
\hline ME4 & 30.9144 & 40.5572 & 39.7137 & 39.8797 & 39.6480 & 38.4851 \\
\hline UQI & 0.4277 & 0.4703 & 0.4632 & 0.5602 & 0.5596 & 0.5545 \\
\hline SSIM & 0.6554 & 0.6080 & 0.6251 & 0.6489 & 0.6477 & 0.6679 \\
\hline AD & 0.1477 & 0.2088 & 0.0587 & 0.07450 & 0.1698 & 0.1045 \\
\hline SC & 0.9841 & 0.8685 & 0.8793 & 0.8705 & 0.8741 & 0.8934 \\
\hline NK & 0.9651 & 0.9840 & 0.9847 & 0.9892 & 0.9871 & 0.9858 \\
\hline MD & 172 & 184 & 183 & 170 & 178 & 173 \\
\hline LMSE & 16.6033 & 40.6008 & 36.6506 & 37.8488 & 37.4703 & 32.2299 \\
\hline NAE & 0.2445 & 0.3661 & 0.3416 & 0.3405 & 0.3408 & 0.3082 \\
\hline
\end{tabular}




\subsection{Denoising using DTDWT and DTCDWT}

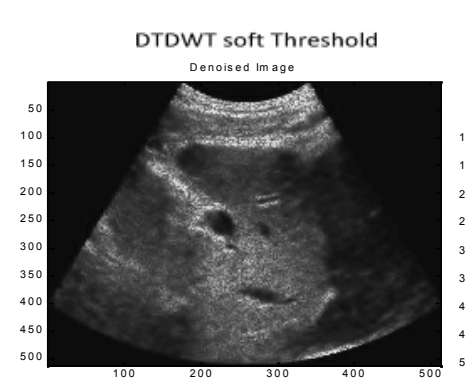

DTCDWT soft Threshold

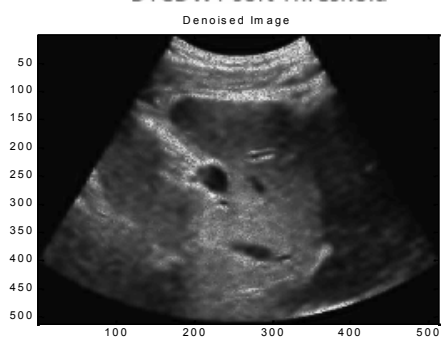

DTDWT hard Threshold

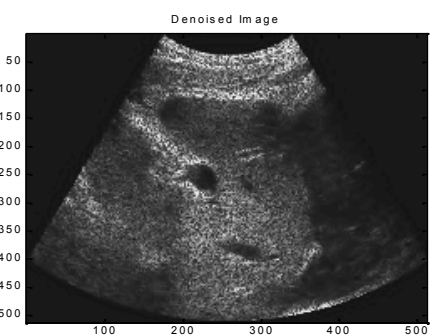

DTCDWT hard Threshold

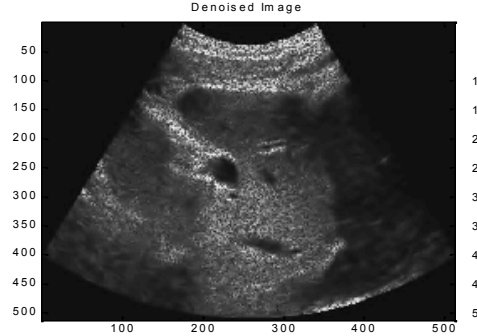

DTDWT semisoft Threshold

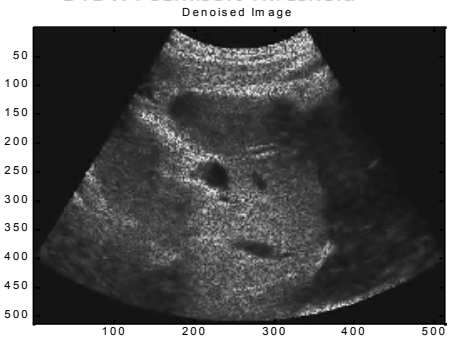

DTCDWT semisoft Threshold

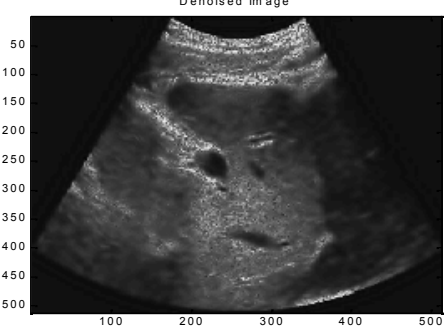

Figure 15: Denoised Results a) DTDWT Hard b) DTDWT Soft c) DTDWT Semi soft d)DTCDWT Hard e) DTCDWT Hard f) DTCDWT semisoft

Table 7: Performance evaluation of DTDWT and DTCWT denoising

\begin{tabular}{|c|l|l|l|l|l|r|}
\hline & \multicolumn{3}{|c|}{ DTDWT } & \multicolumn{3}{c|}{ DTCWT } \\
\hline & Soft & Hard & Semisoft & Soft & Hard & semisoft \\
\hline MSE & 174.8446 & 474.9779636 & 363.2638 & 109.0022152 & 306.5883 & 186.44365 \\
\hline SNR & 15.59092 & 11.52786132 & 12.59465 & 17.57803756 & 13.27528 & 15.324816 \\
\hline RMSE & 13.22288 & 21.79398916 & 19.05948 & 10.4404126 & 17.50966 & 13.654437 \\
\hline PSNR & 28.2959 & 23.95569082 & 25.1202 & 30.34807219 & 25.85687 & 28.016949 \\
\hline ME & 19.43731 & 30.82720132 & 27.86672 & 15.08346778 & 26.3503 & 20.943061 \\
\hline ME4 & 25.60627 & 38.75429771 & 35.83611 & 20.06116653 & 34.50805 & 28.236071 \\
\hline UQI & 0.381646 & 0.362883509 & 0.367974 & 0.356991987 & 0.358108 & 0.3542861 \\
\hline SSIM & 0.70741 & 0.648049385 & 0.674609 & 0.715624647 & 0.688939 & 0.706177 \\
\hline AD & 0.236018 & 0.080404611 & 0.134772 & 0.156454419 & 0.173869 & 0.2438415 \\
\hline SC & 1.008159 & 0.890338178 & 0.929432 & 1.03918384 & 0.953005 & 1.0021953 \\
\hline NK & 0.968465 & 0.986910542 & 0.980852 & 0.96401 & 0.976456 & 0.969593 \\
\hline MD & 143.0497 & 174.5644035 & 167.4451 & 126.6688905 & 180.0035 & 157.7031 \\
\hline LMSE & 9.207953 & 32.32589057 & 23.42276 & 3.172047068 & 19.23603 & 9.7744428 \\
\hline NAE & 0.195119 & 0.313594234 & 0.265771 & 0.163934328 & 0.237563 & 0.1924616 \\
\hline
\end{tabular}




\subsection{Denoising using Double Density and Double Density Dual tree wavelets}

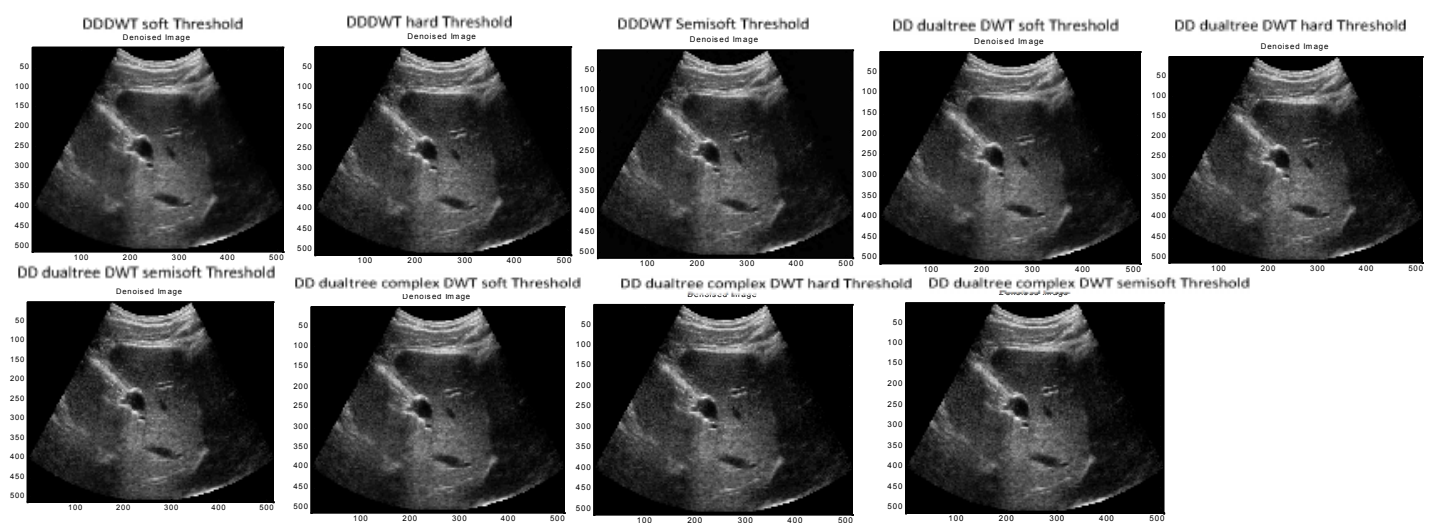

Figure 16: Denoised Results a) Double density Hard b) Double density Soft c) Double density Semi soft d) Double density dual tree Real Hard e) Double density dual tree real Hard f) Double density dual tree real semisoft g) Double density dual tree complex Hard h) Double density dual tree complex soft

i) Double density dual tree complex Semisoft

Table 8: Performance evaluation of Double density wavelets and double density dual tree wavelet denoising

\begin{tabular}{|c|l|l|l|l|l|l|l|l|l|}
\hline & \multicolumn{3}{|c|}{ Double Density DWT } & \multicolumn{2}{c|}{ Double Density Dual Tree DWT } & \multicolumn{3}{c|}{ Double density DTCDWT } \\
\hline & Soft & Hard & Semisoft & Soft & Hard & Semisoft & Soft & Hard & Semisoft \\
\hline MSE & 11.2930 & 1.9521 & 3.4126 & 2.9842 & 0.2215 & 0.4373 & 1.1005 & 0.0269 & 0.0701 \\
\hline SNR & 27.4658 & 35.1253 & 32.6953 & 33.2632 & 44.5794 & 41.6245 & 37.6044 & 53.7333 & 49.5720 \\
\hline RMSE & 3.3605 & 1.3972 & 1.8473 & 1.7274 & 0.4706 & 0.6613 & 1.0490 & 0.1640 & 0.2649 \\
\hline PSNR & 40.1943 & 47.8171 & 45.3915 & 45.9741 & 57.2677 & 54.3139 & 50.3062 & 66.4208 & 62.2598 \\
\hline ME & 4.1427 & 1.7571 & 2.3038 & 2.1381 & 0.5962 & 0.8346 & 1.2958 & 0.2196 & 0.3482 \\
\hline ME4 & 4.7678 & 2.0574 & 2.6768 & 2.4689 & 0.7035 & 0.9816 & 1.4933 & 0.2713 & 0.4240 \\
\hline UQI & 0.6205 & 0.6933 & 0.6783 & 0.6587 & 0.6822 & 0.6795 & 0.6744 & 0.6846 & 0.6839 \\
\hline SSIM & 0.9420 & 0.9880 & 0.9792 & 0.9843 & 0.9985 & 0.9972 & 0.9941 & 0.9997 & 0.9994 \\
\hline AD & $-2.05 \mathrm{E}-$ & $-2.05 \mathrm{E}-12$ & $-2.05 \mathrm{E}-$ & $3.13 \mathrm{E}-$ & $3.14 \mathrm{E}-$ & & $3.13 \mathrm{E}-$ & $3.13 \mathrm{E}-$ & \\
\hline SC & 1.0191 & 1.0020 & 1.0041 & 1.0108 & 1.0004 & 1.0009 & 1.0066 & 1.0000 & 1.0002 \\
\hline NK & 0.9888 & 0.9986 & 0.9974 & 0.9941 & 0.9997 & 0.9994 & 0.9965 & 0.9999 & 0.9998 \\
\hline MD & 18.6064 & 10.2349 & 10.0156 & 10.2421 & 3.3917 & 4.4710 & 5.9894 & 1.7016 & 2.6631 \\
\hline LMSE & 0.2319 & 0.1022 & 0.1390 & 0.08409 & 0.0167 & 0.0286 & 0.0350 & 0.0026 & 0.0062 \\
\hline NAE & 0.0614 & 0.0250 & 0.0338 & 0.0314 & 0.0083 & 0.0118 & 0.0191 & 0.0027 & 0.0045 \\
\hline
\end{tabular}

\section{CONCLUSIONS AND FUTURE WORK}

The development and implementation of denoising filters in spatial domain and using the multiscale transforms are carried out in this paper. The denoising results shows that the spatial filters are smoothing the edges and lines in the images. The wavelet transform based techniques are minimizing the smoothing but failed to differentiate the directional edges. The performance of the undecimated wavelet transform is good but the computational cost will increase with the increase in number of levels. The dual tree complex wavelet transforms and the double density dual tree complex wavelet transforms are outperforming and removing the speckle noise effectively compared to the spatial filtering and other multiscale transforms without increasing the computational cost. This is due to their over completeness. The denoising procedures developed here are considered only global threshold and same shrinkage rule over entire subbands. The denoising efficiency can be improved by developing the threshold calculation and shrinkage rules that are adaptive to level by level or subband by subband. 


\section{REFERENCES}

[1] J.W. Goodman, "Some fundamental properties of speckle," J. Opt. Soc. Am., vol. 66, no. 11, pp. 1145-1149, 1976.

[2] C.B. Burckhardt, "Speckle in ultrasound B-mode scans," IEEE Trans. Sonics Ultrasonics, vol. SU-25, no. 1, pp. $1-6,1978$.

[3] Z. Tao, H. D. Tagare, and J. D. Beaty, "Evaluation of four probability distribution models for speckle in clinical cardiac ultrasound images." IEEE Transactions on Medical Imaging, 25(11):1483-1491, 2006.

[4] P. C. Tay, S. T. Acton, and J. A. Hossack, "A stochastic approach to ultrasound despeckling." In Biomedical Imaging: Nano to Macro, 2006. 3rd IEEE International Symposium on, pages 221-224, 2006.

[5] J.S. Lee, "Digital image enhancement and noise filtering by using local statistics," IEEE Trans. Pattern Anal. Mach. Intell., PAMI-2, no. 2, pp. 165-168, 1980.

[6] J.S. Lee, "Speckle analysis and smoothing of synthetic aperture radar images," Comp. Graphics Image Process., vol. 17, pp. 24-32, 1981, doi:10.1016/S0146-664X(81)80005-6.

[7] J.S. Lee, "Refined filtering of image noise using local statistics," Comput. Graphics Image Process, vol. 15, pp. 380-389, 1981.

[8] V.S. Frost, J.A. Stiles, K.S. Shanmungan, and J.C. Holtzman, "A model for radar images and its application for adaptive digital filtering of multiplicative noise," IEEE Trans. Pattern Anal. Mach. Intell., vol. 4, no. 2, pp. $157-$ $165,1982$.

[9] D.T. Kuan and A.A. Sawchuk, "Adaptive noise smoothing filter for images with signal dependent noise," IEEE Trans. Pattern Anal. Mach. Intell., vol. PAMI-7, no. 2, pp. 165-177, 1985.

[10] D.T. Kuan, A.A. Sawchuk, T.C. Strand, and P. Chavel, "Adaptive restoration of images with speckle," IEEE Trans. Acoust., vol. ASSP-35, pp. 373-383, 1987, doi:10.1109/TASSP.1987.1165131.

[11] J. Saniie, T. Wang, and N. Bilgutay, "Analysis of homomorphic processing for ultrasonic grain signal characterization," IEEE Trans. Ultrason. Ferroelectr. Freq. Control, vol. 3, pp. 365-375, 1989, doi:10.1109/58.19177.

[12] A. Pizurica, A. M.Wink, E. Vansteenkiste, W. Philips, and J. Roerdink, "A review of wavelet denoising in mri and ultrasound brain imaging,” Curr. Med. Imag. Rev., vol. 2, no. 2, pp. 247-260, 2006.

[13] D.L. Donoho, "Denoising by soft thresholding," IEEE Trans. Inform. Theory, vol. 41, pp. 613-627, 1995.

[14] X. Zong, A. Laine, and E. Geiser, "Speckle reduction and contrast enhancement of echocardiograms via multiscale nonlinear processing,” IEEE Trans. Med. Imaging, vol. 17, no. 4, pp. 532-540, 1998.

[15] X. Hao, S. Gao, and X. Gao, "A novel multiscale nonlinear thresholding method for ultrasonic speckle suppressing,” IEEE Trans. Med. Imaging, vol. 18, no. 9, pp. 787-794, 1999.

[16] F.N.S Medeiros, N.D.A. Mascarenhas, R.C.P Marques, and C.M. Laprano, "Edge preserving wavelet speckle filtering," in 5th IEEE Southwest Symposium on Image Analysis and Interpretation, Santa Fe, NM, pp. 281-285, April 7-9, 2002, doi:10.1109/IAI.2002.999933.

[17] C. M. Sehgal, "Quantitative relationship between tissue composition and scattering of ultrasound", J.Acoust. Soc. Am., vol. 94, No.3, pp.1944-1952, Oct.1993.

[18] J. T. M. Verhoeven and J. M. Thijssen, "Improvement of lesion detectability by speckle reduction filtering: A quantitative study", Ultrason. Imag., vol. 15, pp.181-204, 1993.

[19] Paul Butler, "Applied Radiological Imaging for Medical Students", Ist Edition, Cambridge University Press, 2007.

[20] Rangaraj M. Rangayyan, "Biomedical Signal Analysis A Case study Approach”, IEEE Press, 2005.

[21] Stephane Mallat, "A Wavelet Tour of signal Processing", Elsevier, 2006.

[22] D L Donoho and M. Jhonstone, "Wavelet shrinkage: Asymptopia? ”, J.Roy.Stat.Soc., SerB, Vol.57, pp. 301-369, 1995.

[23] D L Donoho, "De-Noising by Soft-Thresholding”, IEEE Transactions on Information Theory, vol.41, No.3, May 1995.

[24] David L. Donoho and Iain M. Johnston, “Adapting to unknown smoothness via wavelet shrinkage”, Journal of the American Statistical Association, vol.90, no432, pp.1200-1224, December 1995. National Laboratory, July 27, 2001.

[25] R. Coifman and D. Donoho, "Translation invariant de-noising," in Lecture Notes in Statistics: Wavelets and Statistics, vol. New York: Springer-Verlag, pp. 125--150, 1995.

[26] S. G. Mallat and W. L. Hwang, "Singularity detection and processing with wavelets," IEEE Trans. Inform. Theory, vol. 38, pp. 617-643, Mar. 1992.

[27] I.Daubechies, "Ten Lectures on Wavelets", SIAM Publishers, 1992.

[28] Z. Wang, A. C. Bovik, H. R. Sheikh and E. P. Simoncelli, "Image quality assessment: From error visibility to structural similarity," IEEE Transactions on Image Processing, vol. 13, no. 4, pp. 600-612, Apr. 2004.

[29] Ivan W.Selesnick, Richard G.Baraniuk, Nick G. Kingsbury, "The Dual Tree Complex Wavelet Transform”, IEEE Signal Processing Magazine, November 2005.

[30] I. W. Selesnick, "The design of Hilbert transform pairs of wavelet bases via the flat delay filter," in Proc. IEEE Int. Conf. Acoust., Speech, Signal Process., May 2001. 
[31] N. G. Kingsbury, "The dual-tree complex wavelet transform: A new technique for shift invariance and directional filters," in Proc. Eighth IEEE DSP Workshop, Salt Lake City, UT, Aug. 9-12, 1998.

[32] I.W.Selesnick, "The double density dual tree DWT", IEEE Transactions on Signal Processing, Vol 52. No.5, May 2004.

[33] I. W. Selesnick, "Sparse signal representations using the tunable Q-factor wavelet transform." In Proc. SPIE 8138 (Wavelets and Sparsity XIV), August 2011.

[34] I. W. Selesnick, "Wavelet Transform with Tunable Q-Factor", IEEE Trans. on Signal Processing. 59(8):35603575, August 2011.

[35] I. W. Selesnick and O. G. Guleryuz, “A diagonally-oriented DCT-like 2D block transform.” In Proc. SPIE 8138 (Wavelets and Sparsity XIV), August 2011.

[36] I. Bayram and I. W. Selesnick, "A subband adaptive iterative shrinkage/thresholding algorithm." IEEE Trans. on Signal Processing. 58(3):1131-1143, March 2010.

[37] I. Bayram and I. W. Selesnick, "On the frame bounds of iterated filter banks." Applied and Computational Harmonic Analysis. 27(2):255-262, September 2009.

[38] A. N. Akansu and W. A. Serdijn and I. W. Selesnick, "Emerging applications of wavelets: A review." Physical Communication. 2009. doi:10.1016/j.phycom.2009.07.001.

[39] I. W. Selesnick and M. A. T. Figueiredo, "Signal restoration with overcomplete wavelet transforms: comparison of analysis and synthesis priors." In Proceedings of SPIE, volume 7446 (Wavelets XIII), August 2-4, 2009.

[40] I. Bayram and I. W. Selesnick, "On the dual-tree complex wavelet packet and M-band transforms." IEEE Trans. on Signal Processing, 56(6):2298-2310, June 2008. Software (zip file).

[41] B. Dumitrescu, I. Bayram, and I. W. Selesnick, "Optimization of symmetric self-Hilbertian filters for the dualtree complex wavelet transform." IEEE Signal Processing Letters, 15:146-149, January 1, 2008.

[42] I. W. Selesnick, "Wavelets, a modern tool for signal processing." Physics Today. 60(10):78-79, October 2007.

[43] A. F. Abdelnour and I. W. Selesnick, "Symmetric nearly shift-invariant tight frame wavelets." IEEE Trans. on Signal Processing, 53(1):231-239, January 2005.

[44] A. F. Abdelnour and I. W. Selesnick, "Symmetric nearly orthogonal and orthgonal nearly symmetric wavelets.”The Arabian Journal for Science and Engineering, vol. 29, num. 2C, pp:3-16, December 2004. 